\title{
Role of oxygen derived free radicals in platelet activating factor induced bowel necrosis
}

\author{
J P CUEVA AND WEI HSUEH
}

From the Department of Pathology, Children's Memorial Hospital, Northwestern University Medical School, Chicago, Il 60614, USA

SUMmary The mechanism of tissue and cell injury in ischaemic bowel necrosis is unclear. The present study investigated the role of oxygen derived free radicals in the development of bowel necrosis using injections of platelet activating factor (PAF) into the mesenteric vasculature. Animals were pretreated with allopurinol or superoxide dismutase together with catalase, before administration of PAF. Superoxide dismutase/catalase markedly improved the PAF-induced lesions, indicating that most of the intestinal damage after PAF injection is because of the release of oxygen radicals. The major source of oxygen radicals is xanthine oxidase, as allopurinol ameliorated small bowel lesions. Pretreatment with allopurinol produced a significant $(\mathrm{p}<0 \cdot 01)$ preventive effect on PAF induced hypotension. In contrast, superoxide dismutase/catalase did not alter PAF induced hypotension. Superoxide dismutase/catalase pretreatment improved PAF induced haemoconcentration and leucopenia, while allopurinol showed no effect.

Platelet activating factor (PAF), also named AGEPC ${ }^{1}$ or PAF acether, ${ }^{2}$ is an endogenous phospholipid mediator which is produced by a wide variety of cells ${ }^{34}$ and mediates a myriad of biological responses. ${ }^{12}$ Platelet activating factor is produced by ischaemic intestinal tissue ${ }^{5}$ and our previous work has shown that injection of PAF in rats produced necrosis of the intestinal tract. ${ }^{6}$ The lesions in our experimental model are morphologically indistinguishable from those of human ischaemic bowel necrosis or necrotising enterocolitis. The pathogenesis of bowel necrosis after PAF injection is probably not a result of thromboembolic phenomen ${ }^{6}$ but rather of release of secondary mediators, ${ }^{7-9}$ resulting in mesenteric vasoconstriction and subsequent ischaemia. The mechanism of tissue injury and cell necrosis, however, remains unclear. Recent evidence suggests that oxygen derived free radicals may be abundantly produced in ischaemic tissues, accounting for at least part of the damage observed. ${ }^{10-15}$ The major source of oxygen radicals in postischaemic tissues appears to be the enzyme xanthine oxidase, ${ }^{12-15}$ high levels being found in intestinal tissue. ${ }^{16} 17$ It is probable that

Address for correspondence: Dr Wei Hsueh, Department of Pathology, Children's Memorial Hospital, 2300 Children's Plaza, Chicago, Il 60614, USA.

Received for publication 11 March 1988. oxygen radicals are generated during the postischaemic reperfusion period after PAF administration and play an important role in the development of bowel necrosis. In the present study, we investigated any attenuating effects of the ubiquitous superoxide dismutase/catalase and allopurinol (a xanthine oxidase inhibitor) on PAF induced bowel necrosis. Superoxide dismutase metabolises superoxide anions and catalase converts subsequently formed hydrogen peroxide to more innocuous substances.

Methods

RATS

Male Sprague-Dawley rats weighing $275( \pm 50)$ $g$ were anaesthetised with pentobarbital ip. The carotid artery and jugular vein were cannulated for blood pressure monitoring, drug administration, and blood sampling as previously described. ${ }^{6}$ PAF, (1-0-alkyl-2-0- acetyl- sn-glycero-3- phosphocholine, Calbiochem-Behring, La Jolla, CA, USA) was diluted from a stock solution in ethanol and stored at $-70^{\circ} \mathrm{C}$. Working solutions were prepared in 2.5 $\mathrm{mg} / \mathrm{ml}$ bovine serum albumin saline solution. Four different pretreatment regimens were assigned to animal groups as follows: (a) pretreatment with $10 \mathrm{mg} / \mathrm{kg}$ superoxide dismutase and $10 \mathrm{mg} / \mathrm{kg}$ catalase 
(Sigma, St Louis, Mo, USA), dissolved in $0.9 \%$ saline, continuously infused at the rate of 0.01 $\mathrm{ml} / \mathrm{min}$, starting at $30 \mathrm{~min}$ before PAF administration, and continued for 150 minutes. (b) Infusion of vehicle (saline) only. (c) Intraperitoneal injection of $5 \mathrm{mg} / \mathrm{kg}$ allopurinol (Sigma), dissolved in dimethylsulphoxide (DMSO) $(2.5 \mathrm{mg} / \mathrm{ml}), 30 \mathrm{~min}$ before PAF injection. (d) Injection of vehicle (DMSO) only. All animals received PAF $(4 \mu \mathrm{g} / \mathrm{kg})$ through aortic injection, proximal to the superior mesenteric artery, as previously described. ${ }^{6}$ The abdominal incision was covered with gauze moistened with warm saline, and the bowel was gently pulled out for examination with the aid of a Wild stereomicroscope. Blood samples were drawn immediately before PAF administration, and at 15,45 , and 105 , and 150 minutes post-PAF injection.

At the termination of the experimental period the grossly discoloured bowel was examined and total circulatory supravital staining done by instillation of

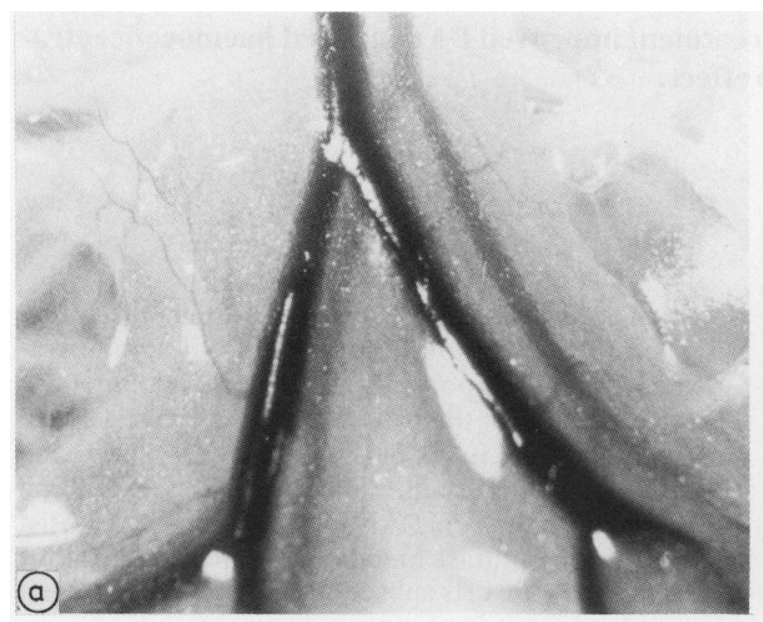

Fig. 1 PAF induced an initial phase of vasoconstriction, followed by a recovery phase. (a) Before PAF injection. Note the diameters of normal mesenteric vessels. (b) 30 min after $P A F$ injection $(4 \mu \mathrm{g} / \mathrm{kg})$. Note vasoconstriction of the same mesenteric artery. (c) 150 min after PAF injection. Note recovery of the vasomotor tone of the same mesenteric artery. Similar changes were observed in serosal vessels (not shown).
$2 \mathrm{ml} 5 \%$ Evans blue. The length of non-stained bowel was recorded and sections were taken for histological examination to confirm the presence of necrosis. Histological assessment of intestinal injury utilised a 0 to 4 grading scale: 0 having normal histology, 1 showing epithelial cell loss injury at villus tips, 2 including loss of more than half of villi, 3 being damage extending to submucosa, and 4 having transmural necrosis.

\section{Results}

Within a few minutes after PAF injection $(4 \mu \mathrm{g} / \mathrm{kg})$, a vasoconstriction response of the small mesenteric vessels in the mesentery itself and the vessels on the serosal surface of the bowel developed (Fig. 1). Five to 10 minutes after PAF administration, the normal mesenteric pulsations totally stopped and many small serosal vessels became undiscernible. As early as 45 minutes, the mesenteric vessels began to recover
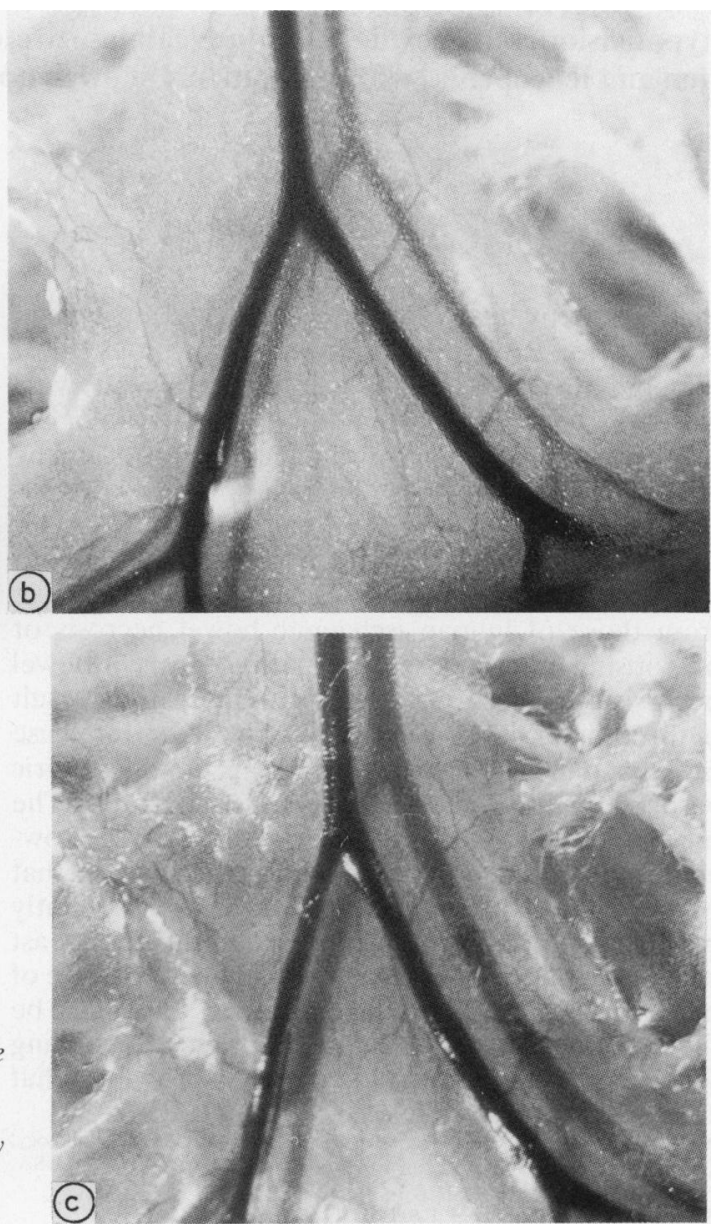

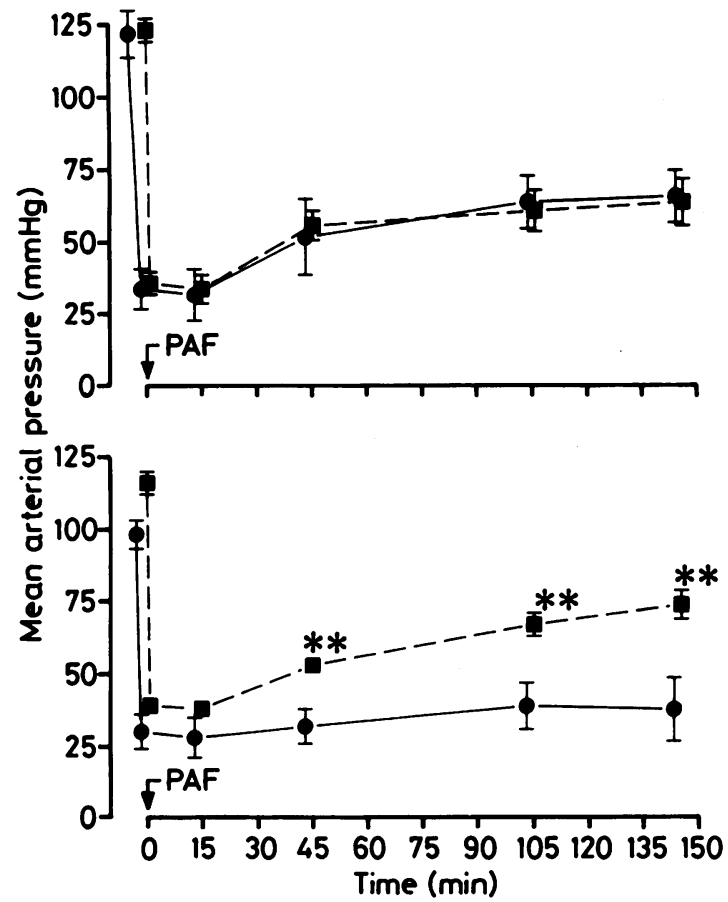

Fig. 2 Effects of superoxide dismutase/catalase and allopurinol on PAF induced systemic hypotension. Upper panel: $\quad$ A bolus dose of PAF $(4 \mu \mathrm{g} / \mathrm{kg})$ and vehicle (saline) only, injected into the aorta at time $0(n=5)$.

- - : pretreatment with $10 \mathrm{mg} / \mathrm{kg}$ superoxide dismutase $+10 \mathrm{mg} / \mathrm{kg}$ catalase, starting at $30 \mathrm{~min}$ before PAF injection, $0.01 \mathrm{ml} / \mathrm{min}$, and continued until the end of the experimental period. $P A F$ was injected at time $0(n=10)$. Lower panel: : PAF+vehicle (DMSO) only $(n=5)$.

-D: Pretreatment with intraperitoneal injection of allopurinol (5 $\mathrm{mg} / \mathrm{kg}$ ), followed by PAF injection (time 0) $(n=15)$. Mean $(S E){ }^{*}=p<0 \cdot 05{ }^{* *}=p<0 \cdot 01$.

from this constricting response, and between 100 and 150 minutes, most vessels showed partial recovery (Fig. 1). Some visible arterial pulsation also returned. Thus we conclude that the bowel was reperfused after an initial vasoconstriction phase after PAF injection.

The systemic effects of PAF $(4 \mu \mathrm{g} / \mathrm{kg})$ include an immediate drop in mean arterial pressure. This severe initial hypotension was observed in all groups of animals (Fig. 2). The initial severe hypotensive episode (approximately 15-30 minutes) was followed by a gradual rise, which eventually (at 150 minutes) reached $48,45,61 \%$, and $36 \%$ of the original blood pressures in groups (a), (b), (c), and (d) respectively. The group receiving allopurinol pretreatment showed significant $(p<0.01)$ recuperation of blood pressure after PAF administration in comparison with the other groups. The WBC count decreased an average of $44 \% 15$ minutes after PAF injection and remained
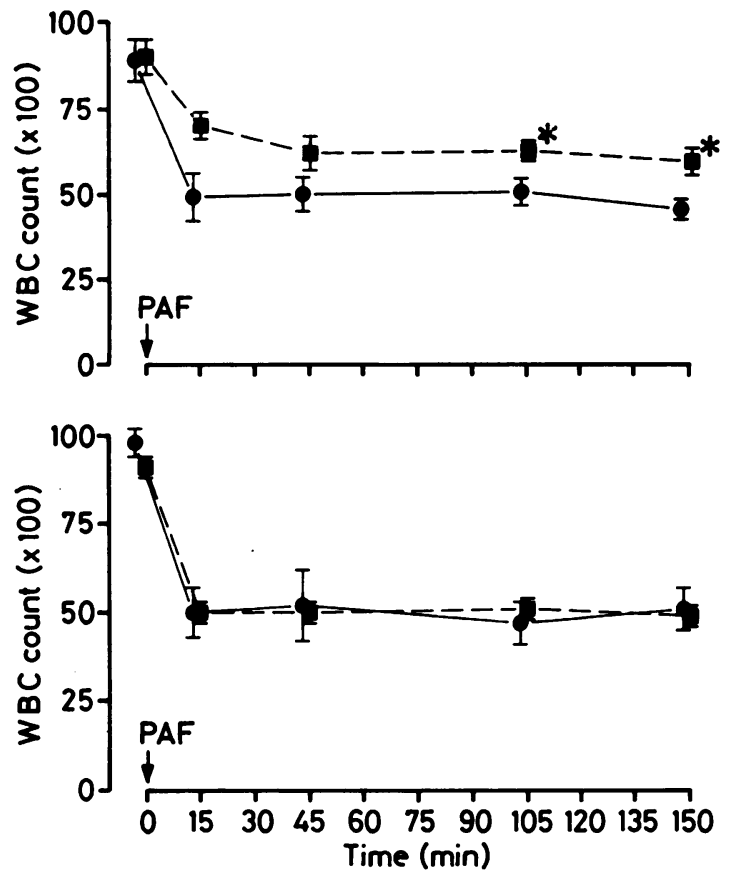

Fig. 3 Effects of superoxide dismutase/catalase (upper) and allopurinol (lower) on PAF induced leukopenia. See Fig. 2 legend for symbols and numbers of experiments. Mean (SE).

low in groups (b), (c), and (d) thereafter (Fig. 3). Interestingly, the group receiving superoxide dismutase/catalase (a) had a significantly $(p<0.05)$ milder leucopenia. All groups also developed marked increase in haematocrit (Hct) 15 minutes after PAF administration which remained high in groups (b), (c), and (d) (Fig. 4). Pretreatment with allopurinol had no effect on PAF induced haemoconcentration while superoxide dismutase/catalase pretreatment (group a) significantly ameliorated the haemoconcentration (Fig. 4). Intraperitoneal injection of pentobarbital on sham operated controls had no significant effects on blood pressure, haematocrit, WBC count, and intestinal perfusion.

Both superoxide dismutase/catalase and allopurinol significantly $(\mathrm{p}<0.01)$ ameliorated intestinal perfusion (as assessed by supravital staining) and attenuated the necrosis (Fig. 5). Only $40 \%$ of the small bowel showed perfusion when PAF was given alone, while pretreatment with superoxide dismutase/catalase or allopurinol improved perfusion to $70 \%$. The degree of necrosis (histological score) also improved. In most animals injected with PAF alone, necrosis involved most part of mucosa or submucosa, while animals receiving PAF plus a pretreatment regimen (superoxide dismutase/catalase or allo- 

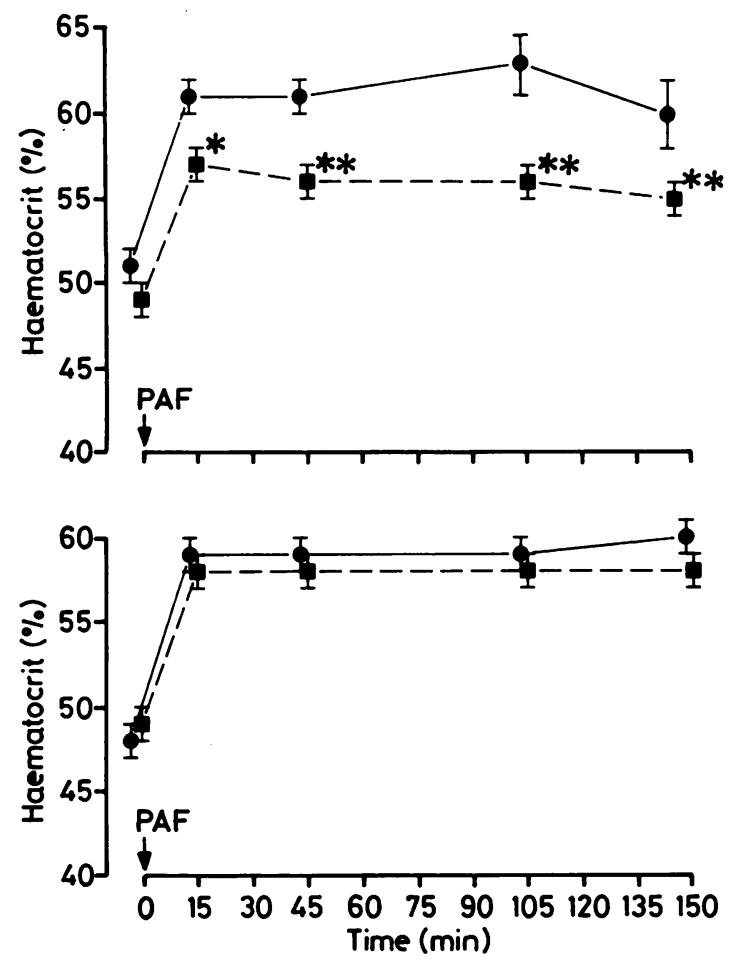

Fig. 4 Effects of superoxide dismutase/catalase and allopurinol on PAF induced haemoconcentration. See Figure 2 legend for symbols and numbers of experiments. Mean (SE).

purinol) developed no or mild necrosis limited to villus tips.

\section{Discussion}

Ischaemic bowel necrosis, or necrotising enterocolitis, is a life threatening condition often associated with shock or infection. We have developed an animal model of ischaemic bowel necrosis associated with shock by injecting PAF into the mesenteric vascular bed. Although evidence has suggested PAF induced bowel necrosis is caused by intestinal ischaemia, probably as a result of vasoconstricting mediators, the cellular mechanism of developing necrosis remains unclear. It has been shown that ischaemic injury in heart, ${ }^{18-20}$ kidney, ${ }^{2122}$ liver,,$^{23}$ and intestine ${ }^{24-27}$ is largely caused by superoxide radicals generated during the reperfusion period. Xanthine oxidase has been proposed by some investigators to play an important role in the reperfusion injury. ${ }^{24}$

In PAF induced intestinal ischaemia, a recovery from the initial vasoconstriction of the small mesen-
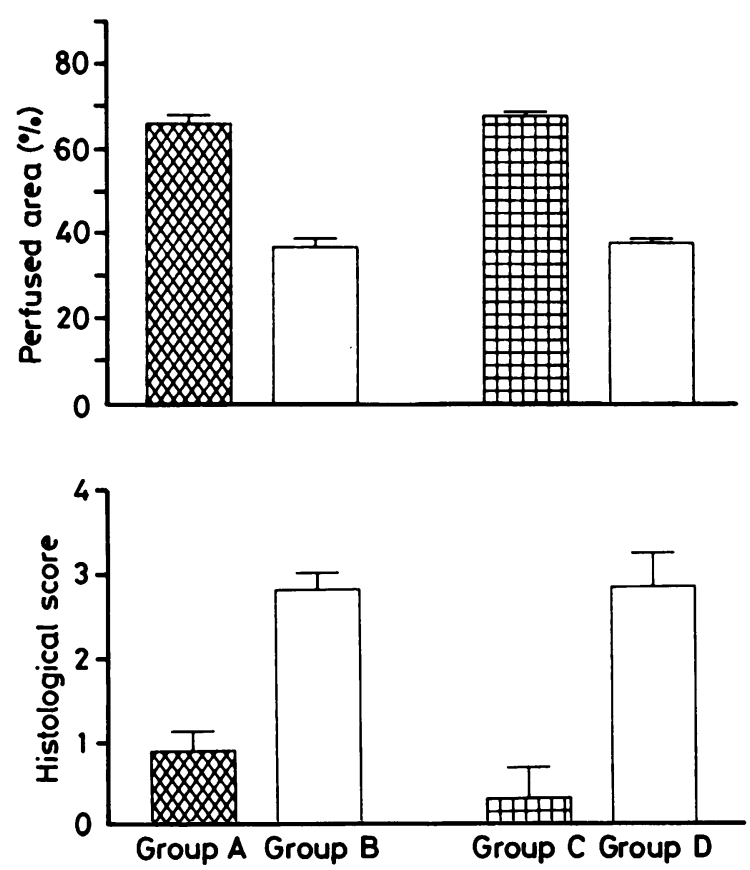

Fig. 5 Effects of superoxide dismutase/catalase and allopurinol on PAF induced bowel necrosis. At the termination of experiment, $5 \%$ Evans blue was injected intravenously, and the length of blue stained bowel was recorded and presented as percentage of entire small intestinal length (upper panel). Sections were taken from the non-stained (non-perfused) areas for histological examination utilising a 0 to 4 grading scale for assessing histological injury. 0: normal, 1: injury at villus tips, 2: loss of more than half of villi, 3: damage extending to submucosa, 4: transmural necrosis (lower panel). Group A: PAF+ pretreatment with superoxide dismutase/catalase and catalase. Group B: PAF+vehicle (saline) only. Group C: $P A F+$ pretreatment with allopurinol. Group D: $P A F+$ vehicle (DMSO) only. See Figure 2 legend for numbers of experiments. Mean (SE).

teric arteries and the intestinal arteries was observed, suggesting reperfusion of the intestinal tissue after the initial vasoconstriction phase. Thus, it is very likely that oxygen radicals are also involved in the pathogenesis of bowel necrosis in our model. The present study has indicated: (a) an important factor of tissue damage and cell necrosis in our model could be accounted for by release of oxygen radicals, as superoxide dismutase/catalase pretreatment markedly ameliorated the necrotic lesions. (b) The major source of oxygen radicals in this model is xanthine oxidase (probably within the intestinal tissue), as allopurinol, a competitive inhibitor of xanthine oxidase, significantly ameliorated PAF induced lesions. (c) The allopurinol group showed significant 
recuperation of blood pressure post-PAF administration. This recuperation is probably caused by inhibition of cardiac xanthine oxidase and the observed severe hypotension is partially attributed to PAFinduced cardiac depression. ${ }^{28}$ Xanthine oxidase has been found in heart tissue, the source appears to be endothelial cells of capillary and small vessels. ${ }^{29}$ (d) Pretreatment with superoxide dismutase/catalase ameliorated PAF induced haemoconcentration, suggesting that oxygen radicals are involved in the development of systemic increase in vascular permeability. (e) Superoxide dismutase/catalase pretreatment also improved PAF induced leucopenia which presumably resulted from lung polymorphonuclear leucocytes sequestration. This observation suggests that oxygen radicals may also be involved in this process, although the detailed mechanism remains unclear. (f) Interestingly, allopurinol had no attenuating effects on PAF-induced haemoconcentration or leucopenia. This observation is different from those of the ischaemia reperfusion models reported by other investigators. ${ }^{14}$ This difference is probably because of the systemic effects of PAF in our model. Platelet activating factor has been shown to activate polymorphonuclear neutrophils (PMN) and induce superoxide release by these cells. ${ }^{30}$ The released radicals and other PMN products subsequently cause endothelial cell damage and an increase in vascular permeability. It may be that systemic effects such as leucopenia and increased vascular permeability in our model are partly accounted for by direct activation of circulating leucocytes by PAF, while much of the intestinal injury observed after PAF administration was a consequence of local formation of xanthine oxidase.

It has been shown that treatment with either allopurinol or superoxide dismutase prevents ischaemia-reperfusion induced neutrophil infiltration in the intestine, ${ }^{31}$ and depletion of granulocytes with neutrophil antiserum or prevention of granulocyte adherence with a monoclonal antibody afforded protection against ischaemia reperfusion injury in the intestine.$^{32}$ It is possible that part of the protective effect of allopurinol and superoxide dismutase was the result of prevention of oxygen radical mediated neutrophil infiltration. We do not believe, however, that neutrophils play an essential role in PAF induced bowel necrosis. This is because (a) there was a marked sequestration of neutrophils in the lung and a severe systemic leucopenia after PAF injection, ${ }^{6}$ and (b) preliminary experiments using Mustargen induced leucopenic rats failed to show any protective effect against PAF induced bowel injury (unpublished observation).

Our previous studies have shown that PAF injection resulted in development of bowel necrosis and this action was potentiated by endotoxin. ${ }^{6}$ The pathogenesis of bowel necrosis seems to involve release of vasoconstrictive mediators, such as peptide leucotrienes and noradrenaline, ${ }^{7-9}$ which results in prolonged intestinal ischaemia. Furthermore, we have recently reported that PAF was formed within bowel tissue in response to lipopolysaccharide. ${ }^{5}$ Based on these previous observations and our present findings, we hypothesise on the pathogenesis of ischaemic bowel necrosis as follows: PAF is formed endogenously as a response to infection or release of endotoxin. The produced PAF releases peptide leucotrienes and other vasoconstrictive mediators in the bowel, which result in prolonged ischaemia and initial small bowel necrosis. The intestine is one of the richest sources of xanthine oxidase in the body ${ }^{16} 17$ and exists as an $\mathrm{NAD}^{+}$reducing, xanthine dehydrogenase. This high activity is found primarily in the mucosal layer, with an increasing gradient of activity from villus base to tip. ${ }^{33}$ This phenomenon may explain our observation that bowel necrosis as a rule begins at the tip of the villus. It has been well established that during ischaemia, xanthine dehydrogenase is converted to xanthine oxidase ${ }^{10-15}$ and ATP is catabolised to hypoxanthine, which then accumulates locally in the tissue. We have also reported that vasodilating prostaglandins (PG) are also released after PAF administration. ${ }^{78}$ The released PGs and other vasodilators resulting in intestinal reperfusion. Reperfusion provides molecular $\mathrm{O}_{2}$ to react with the accumulated tissue hypoxanthine to generate oxygen metabolites. This large burst of oxygen radicals further damages tissue and causes more necrosis.

Our present studies indicate that most of the tissue damage in the bowel is a result of reperfusion injury caused by release of oxygen radicals. PAF, whether endogenously produced or exogenously administered, also acts on leucocytes to produce oxygen radicals. These toxic oxygen metabolites damage endothelial cells and produce increased vascular permeability. Our observations also suggest that oxygen radicals also play a role in PAF induced leucopenia and haemoconcentration. The detailed mechanism, however, awaits further study.

This work was partly supported by NIH grant DK34574.

\section{References}

1 McManus LM, Hanahan DJ, Demopoulos CA, Pinkard RN. Pathobiology of the intravenous infusion of acetyl glyceryl ether phosphorycholine (AGEPC), a synthetic platelet-activating factor (PAF) in the rabbit. J Immunol 1980; 124: 2919-24.

2 Vargaftig BB, Chignard J, Benveniste $\mathbf{J}$, Lefort $\mathbf{J}$, Wal F. Background and present status of research on 
platelet-activating factor (PAF-acether). NY Acad Sci 1981; 370: 119-37.

3 Henson PM, Lynch JM. Cellular origin of PAF. In: Benveniste J, Arnoux B, eds. Platelet-activating factor and structurally related ether-lipids. Amsterdam: Elsevier, 1983: 75-82.

4 Camussi G, Aglietta M, Coda R, Bussolino F, Piacibello $\mathrm{W}$, Tetta C. Release of platelet activating factor and histamine. II. The cellular origin of human PAF: Monocytes, polymorphonuclear neutrophils and basophils. Immunology 1981; 42: 191-9.

5 Hsueh W, Gonzalez-Crussi F, Arroyave JL. Plateletactivating factor is an endogenous mediator in endotoxin induced bowel necrosis. FASEB J 1987; 1: 403-5.

6 Gonzalez-Crussi F, Hsueh W. Experimental model of ischemic bowel necrosis: The role of platelet activating factor and endotoxin. Am J Pathol 1983; 112: 127-35.

7 Hsueh W, Gonzalez-Crussi F, Arroyave JL. Platelet activating factor induced necrotizing enterocolitis; an investigation of secondary mediators in its pathogenesis. Am J Pathol 1986; 122: 231-9.

8 Hsueh W, Gonzalez-Crussi F, Arroyave JL. Release of leukotriene $\mathrm{C}_{4}$ by isolated perfused rat small intestine in response to platelet-activating factor. J Clin Invest 1986; 78: $108-14$

9 Hsueh W, Gonzalez-Crussi F, Arroyave JL. Platelet activating factor and leukotriene $\mathrm{C}$ release catecholamines from isolated, perfused rat small bowel. Fed Proc 1987; 46: 739 .

10 Fridovich I. Biological effects of the superoxide radical. Arch Biochem Biophys 1986; 247: 1-11.

11 Fridovich I. Superoxide radical: an endogenous toxicant. Ann Rev Pharmacol Toxicol 1983; 23: 239-57.

12 McCord JM. Oxygen derived free radicals in postischemic tissue injury. N Engl J Med 1985; 312: 159-63.

13 McCord JM. Oxygen derived radicals: a link between reperfusion injury and inflammation. Fed Proc 1987; 46: 2402-5.

14 Parks DA, Granger DN. Ischemia-induced vascular changes: role of xanthine oxidase and hydroxyl radicals. Am J Physiol 1983; 245: G285-9.

15 Parks DA, Bulkley GB, Granger DN. Role of oxygen derived free radicals in digestive tract disease. Surgery 1983; 94: 415-22.

16 Battelli MG, Della Corte E, Stirpe F. Xanthine oxidase type D (dehydrogenase) in the intestine and other organs of the rat. Biochem J 1972; 126: 747-9.

17 Krenitsky TA, Tuttle JV, Cattau EL, Wang P. A comparison of the distribution and electron acceptor specificities of xanthine oxidase and aldehyde oxidase. Comp Biochem Physiol 1974; 49b: 687-703.

$18 \mathrm{Kim}$ MS, Akera T. $\mathrm{O}_{2}$ free radicals: cause of ischemiareperfusion injury to cardiac $\mathrm{Na}^{+}-\mathrm{K}^{+}$ATPase. Am J Physiol 1986; 252: H252-7.

19 Jackson CV, Mickelson JK, Pope TK, Rao PS,
Lucchesi BR. $\mathrm{O}_{2}$ free radical-mediated myocardial and vascular dysfunction. Am J Physiol 1986; 251: H122531.

20 Jolly SR, Kane NJ, Baile MB, Abrams GD, Lucchesi BR. Canine myocardial reperfusion injury. Its reduction by the combined administration of superoxide dismutase and catalase. Circ Res 1984; 54: 277-85.

21 Koyoma I, Bulkley GB, Williams GM, Im MJ. The role of oxygen free radicals in mediating the reperfusion injury of cold-preserved ischemic kidneys. Transplantation 1985; 40: 590-95.

22 Ouriel K. Smedira NG, Ricotta JJ. Protection of the kidney after temporary ischemia: Free radical scavengers, J Vasc Surg 1985; 2: 49-53.

23 Atalla SL, Toledo-Pereyra LH, MacKenzie GH, Cederna JP. Influence of oxygen-derived free radical scavengers on ischemic livers. Transplantation 1985; 40: $584-7$.

24 Granger DN, Rutili G, McCord JM. Superoxide radicals in feline intestinal ischemia. Gastroenterology 1981; 81: 22-9.

25 Dalsing MC, Grosfeld JL, Shiffler MA, et al. Superoxide dismutase: A cellular protective enzyme in bowel ischemia. J Surg Res 1983; 34: 589-96.

26 Parks DA, Bulkley GB, Granger DN, Hamilton SR, McCord, JM. Ischemic injury in the cat small intestine: role of superoxide radicals. Gastroenterology 1982; 82: 9-15.

27 Schoenberg MH, Muhl E, Sellin D, Younes M, Schildberg FW, Haglund U. Posthypotensive generation of superoxide free radicals - possible role in the pathogenesis of the intestinal mucosal damage. Acta Chir Scand 1984; 150: 301-9.

28 Levi R, Burke JA, Guo ZG, Hattori Y, et al. Acetyl glyceryl ether phosphorycholine (AGEPC). A putative mediator of cardiac anaphylaxis in the guinea pig. Circ Res 1984; 54: 117-24.

29 Jarasch ED, Bruder G, Heid HW. Significance of xanthine oxidase in capillary endothelial cells. Acta Physiol Scand 1986; suppl 548: 39-46.

30 Shaw JO, Pinckard RN, Ferrigni KS, McManus LM, Hanahan DJ. Activation of human neutrophils with 1-0-hexadecyl/octadecyl-2-acetyl-sn-glyceryl-3-phosphorylcholine (platelet activating factor). J Immunol 1981; 127: 1250-5.

31 Grisham MB, Hernandez LA, Granger DN. Xanthine xanthine oxidase activity in hepatic tissue, A new histochemical method. Adv Exp Med Biol 1976; 76A: 605-9.

32 Hernandez LA, Grisham MB, Twohig B, Arfors KE, Harlan JM, Granger DN. Role of neutrophils in ischemia-reperfusion-induced microvascular injury. $\mathrm{Am}$ J Physiol 1987; 253: H699-703.

33 Auscher C, Amory N, Pasquier C, et al. Localization of xanthine oxidase activity in hepatic tissue, a new histochemical method. Adv Exp Med Biol 1976; 76A: 605-9. 